\title{
El uso de indicadores formales en la temática del modo de ser de la vida fáctica en el joven Heidegger
}

\section{The Use of Formal Indicators in the Thematic of the Mode of Being of the Factual Life in the Young Heidegger}

\author{
FELIPE ARÁMBURO MANILLA \\ BENEMÉRITA UNIVERSIDAD AUTÓNOMA DE PUEBLA
}

Recibido: 02/09/2019 Aceptado:17/02/2020

\section{RESUMEN}

La meta del siguiente ensayo es explicar las raíces metódicas que conciernen al uso de los indicadores normales y así vislumbrar el giro que Heidegger le da al concepto de fenómeno en vistas a desarrollar la temática de los modos de ser. La intención no es llevar a cabo una exposición histórica o sistemática acerca de los indicadores formales, menos aún confrontar el pensamiento del Heidegger joven con su maestro Husserl en torno al concepto de fenómeno, sino más bien tomaré pasajes relevantes que iluminen el significado de dichas nociones y así explicar su uso metódico en vistas a esclarecer la conexión interna de los problemas en torno al acceso de la vida fáctica. La problemática se planteará de la siguiente manera: la vida fáctica no se deja determinar a través de rasgos definitorios que nos digan, por ejemplo, qué es algo como lo «humano», sino cómo se lleva a cabo el existir en cada caso, de ahí que ser fácticamente es lo mismo que decir, cómo se realiza en tanto modo de ser «ahí» en cada caso. A partir de dicha problemática se tratará de determinar el tipo de acceso al modo de ser de la vida fáctica y su correspondiente articulación categorial. Los indicadores formales surgen a partir de la necesidad de elaborar dicha articulación categorial que nos permita hablar correctamente del ser de la vida en su facticidad.

$$
\begin{gathered}
\text { PALABRAS CLAVE } \\
\text { MODO, SENTIDO, INDICADOR FORMAL, FACTICIDAD, FENÓMENO }
\end{gathered}
$$




\begin{abstract}
The goal of the following essay is to explain the methodical roots that concern the use of formal indicators and thus glimpse the turn that Heidegger gives to the concept of phenomenon in order to develop the theme of modes of being. The intention is not to carry out a historical or systematic exposition about formal indicators, let alone confront the thought of the young Heidegger with his teacher Husserl about the concept of phenomenon, but rather I will take relevant passages that illuminate the meaning of these notions and thus explain its methodical use in order to clarify the internal connection of the problems around the access of the factual life. The problem will be posed as follows: factual life cannot be determined through defining features that tell us, for example, what is something like the «human», but how it is carried out to exist in each case; being factually is the same as saying, how it is done as a way of being «there» in each case. Based on this problem, it will be an attempt to determine the type of access to the way of being of the factual life and its corresponding categorical articulation. Formal indicators arise from the need to elaborate such categorical articulation that allows us to speak correctly of the being of life in its facticity.
\end{abstract}

KEYWORDS

MODE, MEANING, FORMAL INDICATOR, FACTICITY, PHENOMENON

\title{
INTRODUCCIÓN
}

UNO DE LOS LEGADOS MÁS IMPORTANTES del joven Heidegger (1919/1923) fue su honda preocupación metodológica a la hora de abordar los problemas referentes al acceso correcto al modo de ser de la vida fáctica. Se destaca en esta etapa del pensamiento de Heidegger el replanteamiento del método fenomenológico, el giro que lleva a cabo en torno al concepto de fenómeno y la manera en que los conceptos filosóficos deben ser tratados en lo que él llamará indicadores formales. Sus preocupaciones en torno al método fenomenológico van dirigidas a determinar el acceso a un modo de ser en concreto, aquel que concierne a la vida fáctica. La meta del siguiente ensayo es explicar las raíces metódicas que conciernen al uso de los indicadores formales y así vislumbrar el giro que Heidegger le da al concepto de fenómeno en vistas a desarrollar la temática de los modos de ser. Aclaro que mi intención no es llevar a cabo una exposición histórica o sistemática acerca de los indicadores formales, menos aún confrontar el pensamiento del Heidegger joven con su maestro Husserl en torno al concepto de fenómeno, sino más bien tomaré pasajes relevantes que iluminen el significado de dichas nociones y así explicar su uso metódico en vistas a esclarecer la conexión interna de los problemas en torno al acceso a la vida fáctica. 


\section{EL PROYECTO HERMENÉUTICO FENOMENOLÓGICO DE LA FACTICIDAD}

El proyecto de la llamada hermenéutica fenomenológica de la facticidad es desarrollada por Heidegger a lo largo de sus primeros cursos de Friburgo (1919/23). A partir de dichos cursos se destaca el problema del tipo de acceso y comprensión categorial de la vida. La meta, en este primer pensamiento de Heidegger, sería dilucidar ese modo de ser y el acto de comprensión en el que se realiza. En el Informe Natorp, Heidegger delinea claramente el proyecto «ontológico» sobre el que se basará su pensamiento posterior:

La filosofía trata el problema del ser de la vida fáctica. Desde este punto de vista, la filosofía es ontología fundamental, de tal manera que las ontologías regionales, determinadas individualmente de forma mundana, reciben de la ontología de la facticidad el fundamento y el sentido de sus problemas. La filosofía trata el problema del ser de la vida fáctica y el modo en que este ser es cada vez nombrado e interpretado mediante el discurso [...] investigación que puede definirse como hermenéutica fenomenológica de la facticidad (2002: 46-47 / 2005: 16).

El proyecto del joven Heidegger plantea reconducir la problemática filosófica y ontológica en dirección al problema de la facticidad. La elaboración de dicho proyecto sería una ontología fundamental que articula en el discurso las categorías de la vida y apunta a fundamentar, desde mi punto de vista, una ontología de los modos de ser. Toda interpretación debe saberse conducir respecto a su objeto y así proponer el tipo de acceso correcto, mediante el orden categorial que hagan transparente el modo de ser que se intenta mostrar. La «hermenéutica fenomenológica» de la facticidad posee para el joven Heidegger precisamente la meta de aclarar ese tipo de acceso mediante el uso de indicadores formales además de radicalizar el concepto del fenómeno.

La problemática se planteará de la siguiente manera: la vida fáctica no se deja determinar a través de rasgos definitorios que nos digan, por ejemplo, qué es algo como lo «humano», sino cómo se lleva a cabo el existir en cada caso, de ahí que ser fácticamente es lo mismo que decir, cómo se realiza en tanto modo de ser «ahí» en cada caso. La expresión «modo de ser» equivale no a las variaciones de un concepto primario que engloba dichas variantes para el caso, sino el ser mismo en cada caso, como lo aclara Heidegger en el curso de verano de 1923 Ontología hermenéutica de la facticidad:

Y fáctico, por consiguiente, se llama a algo que «es» articulándose por sí mismo sobre un carácter de ser, el cual es de ese modo. Si se toma el «vivir» por un modo de «ser», entonces «vivir fáctico» quiere decir nuestro propio existir o estar-aquí en cuanto «aquí» en cuanto expresión abierta, por lo que toca al ser, de su carácter de ser (1999: 26 / 1995: 7). 
Podemos concluir diciendo: el carácter de cómo se es, modo de ser, ser en cada caso y ser «ahí», son expresiones que apuntan al mismo fenómeno. $\mathrm{Su}$ uso se refiere estrictamente al ejercicio del caso, de cómo se realiza algo en tanto ser. El problema surge cuando en la labor interpretativa filosofamos sobre la vida y no desde la vida. La vida es normalmente sometida por la filosofía a conceptos que destacan caracteres que ocultan y distorsionan su movilidad. Los conceptos impuestos a la vida tienden a generalizar y homogeneizar su modo de ser, despojándola de la situación concreta donde «en cada caso» ejerce su movilidad. Lo decisivo en el planteamiento heideggeriano es que si queremos comprender la vida tenemos que apropiarnos de la situación en la que se encuentra y dejarla de considerar como un objeto que se puede llegar a ordenar y clasificar. Comprender a la vida desde su «facticidad» significa precisamente que no se va a establecer un concepto de vida genérico, ni tampoco una realidad con propiedades peculiares, sino a comprender la vida de acuerdo a su historia y modo de ejecutarse en cada situación, es decir, pensar a la vida «en sí y desde sí misma». La hermenéutica fenomenológica de la facticidad tiene como propósito permitir el acceso al modo de ser así entendido de la vida en su facticidad, articulando la comprensión que la vida tiene de sí misma desde el mundo circundante donde vive y así establecer desde el discurso sus categorías fundamentales.

En primer lugar, el proyecto es hermenéutico porque se refiere a la autocomprensión que la propia vida tiene de sí misma. Nos separamos así de la postura de un saber sin presupuestos sometida a la transparencia de un sujeto que contempla de manera neutra el objeto. La autocomprensión de la vida fáctica parte, ante todo, de una «situación hermenéutica» en la que la vida se interpreta a sí misma, situación que precede a toda contemplación de objetos. Incluso la misma aprehensión teorética está siempre antecedida por esta situación hermenéutica, está presente en ella y forma parte de ella. La vida misma queda dispuesta a la interpretación, pero además se realiza en ella:

Con respecto a su 'objeto', en cuanto pretendido modo de acceso a él, la hermenéutica indica que dicho objeto tiene un ser que está capacitado para la interpretación y necesitado de ella, que es inherente a ese ser el estar de algún modo yainterpretado [...] En la hermenéutica se configura para el existir una posibilidad de llegar a entenderse y de ser ese entender [...] el interpretar mismo es un cómo posible distintivo del carácter de ser de la facticidad. La interpretación es algo cuyo ser es el del propio vivir fáctico. Si llamamos, aunque sea impropiamente, a la facticidad 'objeto' de la hermenéutica (como las plantas son objeto de la botánica), diremos que ésta, la hermenéutica, se encuentra en su propio objeto (esto es, como si las plantas, lo que son y como son, fueran la botánica (1999: 33-34 / 1995: 15). 
Cualquier punto de vista teorético, no sólo el perteneciente a las ciencias, sino también al de la filosofía, parten de esa comprensión previa de carácter interpretativo que la vida tiene de sí misma o, lo que es lo mismo, la vida comprende preontológicamente su propio ser. Una ontología como ciencia que pretenda acceder al modo de ser de la vida será la condición de posibilidad para toda investigación filosófica. Si tomamos a la filosofía en el sentido antes mencionado, a ella le está reservada la misión de explicitar (tematizar o traer al concepto) un «sentido de ser» apropiado a la interpretación del ser de la vida fáctica, de manera que su «modo de ser» pueda ser mostrado.

El proyecto también es fenomenológico, porque la cuestión central consiste en tener un acceso genuino, directo e inmediato a la vida como fenómeno primario. El tipo de acceso adecuado a ese modo de ser, que articula la comprensión que la vida tiene de sí misma, es el fenomenológico. La fenomenología constituye el método de acceso correcto a la vida y consolida a «la ciencia originaria de la vida en sí y para sí». Hay que desarrollar un método capaz de «intuir» aquel sentido de ser abierto por la vida. La hermenéutica es fenomenológica en la medida en que la comprensión de las situaciones concretas donde se ejecuta la vida, otorga un sentido que no está mediado por otros conceptos y que se da inmediatamente a la intuición. De ahí que Heidegger haya llamado «intuición hermenéutica» al acceso de estas situaciones o contextos de significado donde se lleva a cabo la vida. El tipo de acceso a la vida fáctica es una «intuición originaria» de aquel sentido de ser que la vida ha abierto en cuanto fáctica. Se habla de una intuición originaria en la medida en que el sentido no depende de otra cosa para donarse. Lo importante es que ese sentido de ser no se da mediante hipótesis, ni tampoco mediante ideas impuestas, sino en la «situación hermenéutica» donde la vida se ejecuta de acuerdo con su propia facticidad. La donación del sentido se lleva a cabo en una intuición hermenéutica que cumple el aspecto fenomenológico del proyecto de la hermenéutica de la facticidad, pero ¿cómo apropiarse entonces de esa situación hermenéutica de modo que ganemos el horizonte previo para comprender un sentido de ser? La respuesta estará en el uso de los indicadores formales.

\section{LOS INDICADORES FORMALES EN LA RADICALIZACIÓN DEL CONCEPTO DE FENÓMENO}

Nos enfrentamos ante la problemática en el tipo de acceso al modo de ser de la vida fáctica y su correspondiente articulación categorial. Los indicadores formales surgen a partir de la necesidad de elaborar dicha articulación categorial que nos permita hablar correctamente del ser de la vida en su facticidad. Pero ¿cómo y en qué medida los conceptos en filosofía tienen la capacidad de determinar el sentido de ser manifiesto para el caso de la vida? ¿Hasta qué punto se puede hablar de una «articulación categorial» para un modo de ser 
que encuentra su concreción en cómo ejecuta sus posibilidades y no en qué es en tanto ente? Y así, en la medida en que un modo de ser se resuelve en situaciones concretas de acuerdo con su movilidad, nos enfrentamos ante el reto metódico de determinar el uso de los conceptos en filosofía de manera que no reduzcan, generalicen ni nivelen ese sentido, sino, al contrario, lo traigan a la comprensión de manera genuina y originaria. Los indicadores formales tendrán para Heidegger la función de apuntar al sentido de ser que surge de la vida fáctica de manera que a través de ellos sea posible la articulación de su modo de ser.

Hay aclarar que al hablar indicadores formales estamos ante una cuestión de orden estrictamente metodológico. Con ellos se determina el tipo de acceso a la vida fáctica y la manera de articular en el lenguaje su modo de ser categorialmente. Tomando como guía los pasajes fundamentales para el tema del curso de invierno de 1920/21 Introducción a la fenomenología de la religión, encontramos en primer lugar que el «indicador formal» [Formale Anzeige] es parte de la teoría del método fenomenológico tal como Heidegger lo desarrollará. Los indicadores formales nos conducen a través de un sentido que tiene como fin guiar la explicación fenomenológica, además determinan más que nada el tipo de acceso al fenómeno, tomando en cuenta que la vida fáctica es el fenómeno que ha de ser mostrado y articulado de acuerdo con su modo de ser (2006: 81 / 1995: 55).

Por lo tanto, para elaborar el complejo categorial de la vida fáctica, Heidegger echará mano de los indicadores formales, que, a diferencia de las categorías tradicionales, no imponen un juicio en el momento tratar de articular el ser de un ente, más bien ellos guían la consideración tratada, de modo que no introducen opinión alguna, tampoco implantan juicios preconcebidos que condicionen problema alguno. Los indicadores formales tratan del sentido e «indican» la situación concreta en la que se ejecuta la vida, que no es generalizable según contenidos conceptuales. Precisamente el término «indicación» viene a significar el «anuncio» de una situación concreta. Heidegger precisa que las expresiones: «tiempo», «histórico», «muerte», entre otras, deben ser tratadas como indicadores formales en el sentido antes referido (2006: 81/1995: 55 y 2007b: 354 / 2004: 427). Pero, sobre todo, la expresión «facticidad» es el indicador formal apropiado para mostrar el sentido de ser de la vida, esto es, facticidad no tiene nada que ver con un contenido quiditativo de la vida, sino la expresión de un sentido que determina ese modo de ser. Los indicadores formales, para su uso en filosofía, no serán esquemas de algo realobjetivo, sino indicativos de una «posibilidad de ser». En la medida en que la filosofía llegue a ser ontología fundamental de la facticidad, entonces se puede decir que todos los conceptos en filosofía son formalmente indicativos, anuncian el modo de ser de lo así indicado. Como puede notarse el indicador for- 
mal tiene esta disposición de instalarse en el sentido sin introducir contenido alguno. El indicador formal es, por lo tanto, un medio metódico genuino para adentrarnos al sentido de ser de la vida y su propia movilidad, pero, ¿cómo un indicador formal tiene esta peculiaridad de poder determinar el tipo de acceso a la vida fáctica?

Respecto a lo «formal», Heidegger se remonta a la diferencia husserliana entre generalización y formalización. Según Heidegger fue un acierto de Husserl haber otorgado a la formalización un sentido renovado para la investigación en fenomenología. Husserl señala que la generalización se aplica a determinados campos de la experiencia, donde se asciende de lo concreto a lo general. Por ejemplo, pasamos del rojo a color y de color a cualidad sensible. Sin embargo, no es lo mismo cuando pasamos de la cualidad sensible a «objeto», o, a «esencia», en éste caso lo que llevamos a cabo es una formalización. A lo cual Heidegger agrega: "Cabe preguntarse si la nota 'cualidad sensible' determina en el mismo sentido 'color' que la nota formal 'objeto' a todo objeto cualquiera. Claro que no. Y, sin embargo, la distinción entre generalización y formalización no está del todo clara» (2006: 84 / 1995: 58). Mientras que en la generalización siempre se quiere decir generalización genérica, por estar ligada al sector temático, en cambio en la formalización, la actitud no está ligada a la tematización, sino que es libre respecto a ella y por lo tanto libre de la gradación sin someterse a los géneros y especies dentro de ese campo temático. No se requieren generalidades inferiores para ascender a la generalidad superior de «objeto». Efectivamente, cuando decimos, por ejemplo, «la piedra es un objeto», empleamos una categoría formal que no pertenece al contenido esencial o naturaleza de la cosa. Lo formal no forma parte de la cosa ni forma parte de su contenido, como sí lo es el color rojo para una silla. La formalización estaría libre de toda referencia al contenido material y se apega a estructuras formales que están siempre supuestas en nuestro conocimiento de las cosas.

La generalización es una manera de ordenar y tiene un uso clasificatorio en filosofía, sin embargo, una vez que se ha elegido, hay que mantenerse en todo momento en la línea de sus gradaciones, su uso hace imposible el salto de un campo temático a otro. En cambio, la formalización, que también expresa un orden, lo hace sin mantenerse en una determinada región temática, «no está ligada a un determinado qué del objeto que hay que determinar». Heidegger agrega: «La definición se desvía del contenido temático del objeto, lo considera según su estar dado [cómo está dado]; se define como algo captado, como algo a lo que va la referencia cognoscitiva. El sentido de 'objeto en general' significa sólo el 'a-qué', de la referencia teórica de la actitud» (2006: 86 / 1995: 61). Surge así la pregunta más importante, y en esto Heidegger trata de 
dar un paso más allá que Husserl. Cuando predicamos formalmente ¿a qué motivación se obedece? La respuesta de Heidegger es la siguiente:

[La formalización] brota del sentido de la referencia actitudinal misma. Ya no extraigo el objeto con la mirada la nota del qué de los objetos, sino veo su nota como estando en él. Debo apartar la mirada de su quididad [Wasgehalt] y estar pendiente de que el objeto esté dado y captado actitudinalmente. Así brota la formalización del sentido referencial de la referencia actitudinal misma y no de una quididad en general (2006: 84 / 1995: 58).

La motivación a la que obedece la formalización, y en esto tiene su propio origen, es aquello que Heidegger llama el «sentido de referencia» [Bezugsein]. Por lo tanto, cualquier categoría ontológico-formal (objeto, cosa, esencia, etc.) constitutivas de la actitud teórica, surgen de este sentido de referencia. Heidegger advierte que la actitud teórica, a pesar de estar determinada por el sentido de referencia, no es un ejercicio originario. La determinación de algo en cuanto objeto, esencia, etc., (categorías formales) no corresponden al tratamiento originario de los fenómenos, sino que solo representa una conformación de una referencia. Por esta razón la tarea de la conformación de una pluralidad referencial basado en categorías formales, que impulsa la ontología formal propuesta por Husserl (mathesis universalis) representa un intento parcial e incompleto para el proyecto fenomenológico de Heidegger (2006: 84-87 / 1995: 58-61).

Lo que Heidegger tiene a la vista es una unidad estructural del fenómeno que involucra varios momentos de su sentido. El sentido de referencia aún tiene que ser contemplado como ejercicio si queremos captar el sentido integro del fenómeno. Lo «formal» en el término «indicador formal» tiene el significado para Heidegger de abarcar incluso ese sentido de su ejercicio. Mientras que «formal» para la «ontología de lo formal» en Husserl es algo conformado objetivamente, el anuncio «formal» nada tiene que ver con lo «actitudinalmente teorético», esto es, con una conformación de lo objetivo desde su puro sentido de referencia. La formalización como se dijo no está ligada a un determinado «qué» del objeto, se encuentra libre del contenido. El «sentido de referencia» expresado en la formalización no pertenece al orden temático de una región, es una categoría objetiva formal liberada de contenidos temáticos. Sin embargo, la formalización, según Heidegger, debe tomarse originariamente y adquirir una nueva dimensión, aquella que es tarea para la fenomenología: el sentido de referencia dentro de su ejercicio. Por lo tanto, además del sentido de referencia y el sentido de contenido, tenemos un sentido de ejecución [Vollzugssin] que pertenece también al sentido del fenómeno. El indicador formal no se reduce a la actitud del puro mirar teorético, ni desde el punto de vista de la generalización que busca ordenar y catalogar entes, ni cierta referencia de lo 
formal-objetivo. Indicar formalmente significa seguir una indicación, tomar la dirección de un sentido que se dona, haciendo énfasis en aquel ejercicio donde se lleva a cabo dicha donación. Si los indicadores formales apuntan a la «dirección indicada de sentido», entonces tenemos ahora una nueva manera de entender al fenómeno y la labor de la fenomenología. Los indicadores formales pasan a ser la guía para toda investigación fenomenológica y por lo tanto usados en el acceso al ser de la vida fáctica en tanto fenómeno. El «modo» de ser, sería entonces el concepto que indica la manera en cómo el fenómeno se conforma de acuerdo con un sentido.

\section{LA RADICALIZACIÓN DEL CONCEPTO DE FENÓMENO A PARTIR DE LOS INDI- CADORES FORMALES}

En el curso citado de los Introducción al problema de la religión, Heidegger dice: «En la metodología llamamos 'indicador formal' al empleo de un sentido que guía la explicación fenomenológica. Lo que el sentido formalmente anunciado lleva en sí constituye el horizonte en el que los fenómenos serán avistados» (2006: 81 / 1995: 55). Como se ha dicho, los indicadores formales pertenecen a la «teoría del método fenomenológico mismo» y nos indican cómo los fenómenos son puestos en el horizonte de su sentido. Tenemos así una ampliación de los alcances de la fenomenología que expresa el nuevo y renovado concepto de fenómeno. A partir de la ampliación del significado de lo formal, el planteamiento de Heidegger determina tres direcciones del sentido del fenómeno: a) sentido de contenido [Gehaltssinn]: el «qué» originario que es experimentado, el lado «objetivo» del fenómeno, b) sentido de referencia [Bezugssinn]: el originario «cómo» en que es experiencia, el «hacia qué», c) sentido de ejecución [Vollzugssinn]: el originario «cómo» en que el sentido de referencia es ejercido, o bien, la temporalidad que nos da el cómo se lleva a cabo dicha movilidad y cómo se conserva en esa forma peculiar de movilidad; son tres direcciones de sentido que están unidas en una totalidad. La definición de fenómeno queda establecida como sigue: «una totalidad de sentido según estas tres direcciones», a lo cual agrega Heidegger: «la 'fenomenología' es la explicación de esta totalidad de sentido, da el ' $\lambda$ ó $\gamma o \varsigma^{\prime}$ ' de los fenómenos, ' $\lambda$ ó ${ }_{0} \varsigma^{\prime}$ en el sentido de 'verbum internum' (no en el sentido de la logicización)» (2006: 88 / 1995: 63).

Podemos sostener que Heidegger ha radicalizado el método fenomenológico ya que considera al fenómeno como una unidad de sentido de acuerdo con las tres direcciones antes señaladas: contenido, referencia y ejecución, donde el sentido de ejecución juega el papel preponderante para el método. El «ir a las cosas mismas» como lema de la filosofía constituye para Heidegger, tomar a los fenómenos de acuerdo con dicha unidad estructural de sentido. Como se sabe, para Husserl, el lema «ir a las cosas mismas» expresa un proceder 
descriptivo y no metafísico. Las cosas mismas no son los entes pertenecientes al mundo o la naturaleza, sino el objeto correlativo de determinados actos, aquellos cuyo sentido es presentar a la cosa «en persona» [leibhaftig]; lo que se presenta en dichos actos aparece en una intuición originaria que presenta a la cosa misma, por lo que la fenomenología tiene como meta realizar el acto que da originariamente la objetividad. En este sentido para Husserl, la verdad tiene así el sentido de un cumplimiento de carácter intuitivo [Erfüllung]. Es ilustrativo que Heidegger se apega en muchos aspectos a la idea husserliana de fenomenología, pues ella es: «un cómo de la investigación, aquel que actualiza los objetos de la intuición y sólo habla de ellos en la medida en que están ahí en la intuición. Ese cómo y su realización son algo obvio; por eso, decir 'filosofía fenomenológica' resulta en el fondo equívoco» (2008: 96 / 1995: 72). Sin embargo, la crítica de Heidegger no se centra en la donación de la cosa misma en la intuición, sino más bien en la objetivación que se hace de dicha donación, además Heidegger se separa de esta idea de cumplimiento en la intuición, que reduce la intencionalidad de los actos de conciencia en el sentido de un ver puramente teorético que reduce el aparecer de la cosa a un objeto contemplado por la conciencia. Para Heidegger, la fenomenología trascendental de Husserl tampoco ha determinado el modo de ser de la conciencia, además de haberse dejado llevar por la concepción tradicional del ser como presencia constante sin haberla cuestionado en lo más mínimo. Heidegger se da cuenta que las estructuras de la facticidad no se dejan caracterizar como objetos ahí presentes ante una conciencia que los constituye. Es cierto que Heidegger conserva el término «intuición» para esta captación directa del sentido del fenómeno, el sentido del fenómeno se dona en una intuición, pero a diferencia de una intuición que aísla objetos, es una intuición hermenéutica, que ahora se extiende a los contextos significativos del mundo donde la vida fáctica se mueve.

En el momento en que la fenomenología se ubica en el proyecto de describir las estructuras que dan sentido a la vida fáctica, sufre una transformación radical. Para Heidegger, al resaltar el sentido de ejecución en la estructura de los fenómenos, la fenomenología lleva a cabo el giro correspondiente hacia el «cómo» se nos da algo en su aparecer, donde el qué solamente es accesible a partir del cómo correspondiente del aparecer mismo. La importancia del sentido de ejecución para la determinación del sentido de un fenómeno es que la actitud teorética es insuficiente para mostrar al fenómeno mismo, en todo acto de reflexión el sentido de contenido y el sentido de referencia siempre remitirán previamente al sentido de ejecución. Gracias al sentido de ejecución es como el fenómeno evita ser reducido a la pura esfera temática, hay un «aseguramiento previo de modo que el carácter ejecutivo queda aún libre» (2006: 89 / 1995: 64). Por lo tanto, el sentido de ejecución antecede a todo acto cons- 
ciente reflexivo, de manera que la actitud teorética resulta derivada. Mientras que el sentido de contenido y el sentido de referencia sí están presentes en la mirada teórica, el sentido de ejecución queda oculto, evitando la reducción del fenómeno al puro aparecer objetivo. Por eso Heidegger resaltará el sentido de ejecución del fenómeno, haciendo la aclaración que esta estructura fenoménica ejecutiva no se encuentra separada de los otros sentidos. El indicador es formal porque nos remite al sentido de referencia, tiene que anunciar anticipadamente la referencia del fenómeno. Pero para evitar que la referencia se agote en el objeto y sus caracteres a los cuales se refiere, nos dice Heidegger: «un fenómeno tiene que estar previamente dado de tal modo que su sentido de referencia quede flotando», esto es, que no quede unilateralmente determinado por el objeto y por lo tanto nivelado al ámbito de lo presente en general, tiene que quedar en «suspensión». Sólo en este momento la formalización puede significar un «indicar», donde se pone el énfasis en el sentido de ejecución, esto es, en el ejercicio y no tanto en los contenidos objetivos, evitando una inserción en determinado campo temático. La mirada filosófica no queda atada a la presencialidad del objeto, con el riesgo de identificar al fenómeno con los caracteres objetivos de lo que está-ahí presente, sino más bien queda suspendida como ejercicio libre para la comprensión del fenómeno y así dirigida en vistas al sentido del propio fenómeno.

Como hemos dicho la hermenéutica fenomenológica del primer Heidegger recurre a estos indicadores formales para acceder y articular la comprensión que la vida tiene de sí misma. El ejercicio originario expresado en los indicadores formales es el de la movilidad de la vida en cuanto fáctica; ellos «indican» el modo de ser de la vida, no como su contenido, sino en cuanto exigen de ella una autocomprensión, y sólo en este sentido son «indicativos». El indicador formal no es el concepto cuyo contenido representa a algo presente como propiedad de un ente, lo que indica más bien es el cómo se da algo para la vida misma en tanto modo de ser fundamental y el cómo la vida va madurando su poder-ser constitutivo, de ahí que los indicadores formales son la manera adecuada de expresar su movilidad.

\section{EL SENTIDO FORMALMENTE ANUNCIADO DEL FENÓMENO}

DE LA VIDA FÁCTICA COMO CUIDADO

Pasemos ahora a desarrollar el uso de la indicación formal vista desde la perspectiva del cuidado [Sorge]. La tradición filosófica había determinado y organizado la totalidad del ente a través de sus caracteres esenciales, dicho acto clasificatorio tomó al ente y sus determinaciones como meras generalidades. De acuerdo a este tendencia ontológica a la organización, el sentido de ser pasó a determinarse como la presencia constante del ente, y no es de extrañarse que por esta razón el concepto de substancia haya predominado como el 
sentido de ser primario, sin embargo este sentido de ser como pura presencia es inadecuado para determinar al modo de ser de la vida fáctica, el modo de ser que le corresponde es el del cuidado: la vida está condicionada por el factum de un tener-que-ser, de ocuparse de su propio ser, esto es, cualquiera de los rasgos definitorios de su ser los tiene que ejecutar y llevar a cabo en situaciones concretas. El sentido de ser de la vida como cuidado no puede ser tematizado ni clasificado, tampoco es una esencia dotada de contenido material que se pueda reducir a la pura presencia, más bien dicho sentido tiene que ser determinado a través de los propios actos ejecutivos o posibilidades de ser de la vida. Como lo indica Heidegger, el acceso a la vida tiene que lograrse de acuerdo con una intencionalidad plena donde aparezcan todas las direcciones de sentido del fenómeno desarrolladas en la indicación formal. En un pasaje del Informa Natorp se nos dice que:

La hermenéutica es fenomenológica, lo cual significa que su ámbito objetivo -la vida fáctica en relación con el modo de su ser y de su hablar- se considera según la temática y el método de investigación, como fenómeno. La estructura objetiva que caracteriza a algo como fenómeno, la intencionalidad plena (el estar referido a, el horizonte de referencia como tal, el acto de referirse a, el despliegue temporal de ese acto, la custodia de ese despliegue temporal), no es otra que la del objeto que tiene el carácter ontológico de la vida fáctica. La intencionalidad, entendida pura y simplemente como el estar referido-a, es el primer carácter fenoménico de la actividad fundamental de la vida (es decir, del cuidado) que puede ser puesto inmediatamente de relieve (2002: 47 / 2005: 17).

Lo decisivo en este pasaje es que Heidegger determina metódicamente a la vida fáctica como fenómeno. En primer lugar, la estructura de dicho modo de ser no se agota en las relaciones de intencionalidad de una conciencia objetivamente. Heidegger le llama intencionalidad plena a la intencionalidad que está formada por las tres direcciones de sentido del fenómeno aludidas en la indicación formal, además agrega al sentido de ejecución un carácter temporal y la custodia de ese despliegue temporal. La intencionalidad de la conciencia al estilo husserliano basada en el paradigma de la percepción, donde solamente están considerados los sentidos de contenido y referencialidad acotados a la actitud teórica de la contemplación de objetos, es un caso particular de esta intencionalidad plena [volle Anschauung], que resulta ser «la actividad fundamental de la vida», esto es, su propia movilidad y que ahora Heidegger llamará cuidado [Sorge]. Cualquier comportamiento en el que la vida se desarrolla adquiere su sentido integro y, por lo tanto, su «estructura objetiva», en la intencionalidad plena del fenómeno del cuidado. La fenomenología traspasa la posición contemplativa y teorética a la que la había restringido Husserl. La intencionalidad plena de la que habla Heidegger tiene que ver con considerar íntegramente las estructuras fenoménicas sin estar sometidas al punto 
de vista parcial del ver teorético. La meta será entonces que quede «puesto inmediatamente de relieve» el sentido pleno del fenómeno de la vida fáctica, comprendida ahora como cuidado, de modo que se muestre la modalidad de su ser correspondiente.

El cuidado [Sorge] se convierte en la estructura determinante de la vida fáctica ya que expresa el sentido fundamental de su movilidad y, por la cual, de acuerdo con su estructura intencional, está referida en cada caso a su mundo:

El sentido fundamental de la actividad fáctica de la vida es el cuidado (curare). En el 'estar-ocupado-en-algo' está presente el horizonte dentro del cual se mueve el cuidado de la vida: el mundo que le corresponde en cada ocasión. La actividad del cuidado se caracteriza por el trato que la vida fáctica mantiene con su mundo. El hacia-dónde del cuidado es el con-qué del trato. El significado del ser real y efectivo y el significado de la existencia del mundo se funda y se determina a partir de su carácter: como el asunto mismo del trato propio del cuidado (2002: 35 / 2005: 6).

Para que el cuidado sea el «sentido de la movilidad de la vida», debe expresarse precisamente como unidad estructural de sentido, es decir, ser el sentido integro de la vida sin dejar a un lado cada una de sus direcciones y dimensiones, sólo así otros entes distintos a la vida podrán ser modalizados de acuerdo con las formas del cuidado o comportamientos. Tomando el cuidado como el «sentido de la vida fáctica», como fenómeno primario, resulta ser la base para determinar los caracteres ontológicos del ente, de manera que sean accesibles esos otros modos de ser en tanto fenómenos, es decir, como accesibles para el trato del cuidado. Por ello, no sería aventurado afirmar que el cuidado es el fenómeno por excelencia. Así en la medida en que el cuidado «señala» la especificidad de la movilidad de la vida, expresa también el modo de su «vitalidad» frente a otros modos de ser, llámese «vivos», útiles o cosas. El cuidado destaca íntegramente las direcciones del sentido del fenómeno, que normalmente la actitud teórica nivela u oculta. El cuidado nos da la «cosa misma» del vivir fácticamente, de manera que muestra el modo de ser de la vida fáctica en toda su integridad fenoménica. Además, justo en virtud de esta amplitud de la movilidad del cuidado, es como surgen por un lado distintas maneras de llevarse a cabo su ejecución en comportamientos concretos, pero también surgen distintas referencias con aquello que sale al encuentro en esos comportamientos. En el cuidado están destacadas íntegramente las direcciones de sentido del fenómeno, que la actitud teorética homogeneiza y oculta. Obviamente, el cuidado no es un concepto abstracto que como estructura universal estuviera en la base de todo ocuparse de algo, sino es el sentido de ser que se ejecuta en todo comportamiento concreto en nuestro trato con los entes. Por eso se destaca, como he insistido, el sentido de ejecución, que apunta al cómo 
se lleva a cabo ese modo de ser, y que permite la diversidad y multiplicidad de distintos modos de referencia y, por lo tanto, de contenidos mostrados en esas referencias. Esta es la razón por la que el cuidado no funciona metódicamente como concepto general que engloba genéricamente a los comportamientos, sino que es el indicador formal de la movilidad que expresa el ejercicio especifico del vivir. En la medida en que los comportamientos llevan consigo las direcciones propias del fenómeno, cada comportamiento queda modalizado para expresar el tipo de movilidad específica, ya sea como comportamiento teórico, práctico o instrumental respecto a entes. La concreción de todo ejercicio queda indisolublemente unido a un modo de llevarse a cabo ese comportamiento. Entonces, un análisis riguroso del cuidado, proporciona la clave para el tipo de acceso al modo de ser del ente que se trate. Si recordamos que los comportamientos llevan las direcciones del sentido del cuidado, entonces el cuidado, como indicador formal, anuncia la manera como la vida sale al encuentro de entes. El cuidado muestra la manera como el ente es abierto en su modo de ser correspondiente, de manera que se aclara el tipo de acceso a otros modos de ser distintos a la vida fáctica. En un texto del primer curso de Marburgo, Introducción a la investigación fenomenológica, Heidegger hace una brillante exposición, mejor que ninguna de los cursos de esta época, sobre la estructura del cuidado y su importancia para el acceso a otros modos de ser:

La determinación de los caracteres del ser de un ente se hace posible gracias a la interpretación del cuidado en el que tal ente, como ente determinado, se encuentra. Hemos de decir sobre todo como clave de lo que sigue que la interpretación tiene como tema suyo el modo y la manera del estar ocupado en algo [...] El cuidado no es nada subjetivo y no oculta aquello de lo que se cuida; más bien, lo deja venir a su auténtico ser. Si el ente es preguntando correlativamente al cuidado que lo descubre, entonces lo que debe ser investigado no es el modo de ser entendido, sino precisamente el cómo del venir-al-encuentro-desde-sí-mismo del ente, que se hace posible [...] Únicamente como clave interpretativa respecto del carácter de ser del cuidado hay que decir que, en tanto que cuidado, descubre, antes que nada, aquello de lo que se cuida, y que la ocupación en su específico ser mantiene, como tal el existente por ella descubierto en su modo específico (2008b: 72-73 / 2006:17).

El análisis de la estructura del cuidado promueve una investigación de carácter «ontológico» que pretende establecer el modo de ser de un ente cualquiera. Para lograr el desarrollo de dicha investigación, sólo se requiere que la interpretación recaiga en la manera en que se lleva a cabo el cuidado en sus comportamientos, es decir, en la «manera de estar ocupado de algo». Aquél algo, el ente que sale al encuentro en la ocupación se hace entonces visible en su modo de ser, quedando ya abierto en el cuidado. Esta manera de presentarse del ente a partir del cuidado no tiene nada que ver con una subjetivación, más 
bien el cuidado «abre» al ente tal como es y hace posible acceder a él sin más. A este tipo de acceso al ente a través del ejercicio del cuidado, lo llama Heidegger, descubrimiento. El ente que aparece es «descubierto» en este modo de su presentación. Por lo tanto, lo que hay que investigar es precisamente el cómo del venir al encuentro desde sí mismo del ente, que resulta ser la modalidad específica en la que se ejerce la referencia al ente, y que hace patente al ente en su modo específico de ser. Solo a partir de este enfoque del análisis del cuidado es como la investigación fenomenológica mostrará su relevancia ontológica, es más, la fenomenología no puede llegar al cumplimiento de sus metas si ella no llega a ser ontología. Entonces, si consideramos que la investigación ontológica trata de los modos de ser en general, se nos revela el camino que ha tomado Heidegger, que no es el de una clasificación por la vía categorial de los distintos modos de ser de acuerdo con ciertas regiones del ente predefinidas, dicho punto de vista es un proceder no originario para acceder a los fenómenos. Heidegger exige que se cumpla con aquello que debe ser estudiado mediante el método fenomenológico: «el modo de venir al encuentro desde sí mismo» del ente, de manera que el ente se muestre en sus caracteres mediante el cuidado para que el ente quede desde sí mismo abierto en su ser. Así, el tipo de visión que pertenece al cuidado, aquella que interpretando hace posible el acceso y trato con todo ente posible, permite que el ente abierto desde sí mismo salga a la luz no de manera indiferenciada, con un concepto nivelado de ser como un puro estar-ahí presente, sino de acuerdo con una modalidad de ser correlativa a los comportamientos que ejecuta el cuidado. La hermenéutica fenomenológica de la facticidad tiene su cumplimiento en el análisis de la estructura intencional del cuidado, se trata de un proceder estrictamente fenomenológico e interpretativo que hace comprensible el aparecer de las cosas mismas. El lema originario de la fenomenología del «ir a las cosas mismas desde su aparecer originario» se ha cumplido así cabalmente con la mostración interpretativa del fenómeno del cuidado.

\section{CONCLUSIÓN}

Para concluir me gustaría volver a resaltar la importancia metodológica de los indicadores formales en relación con el acceso de la vida fáctica y sus consecuencias para una ontología general de los modos de ser. Los indicadores formales surgen a partir de la necesidad de elaborar una articulación categorial que nos permita hablar correctamente del ser de la vida en su facticidad; nos permiten desarrollar la propia movilidad de la vida fáctica y orientarnos a través de ella, reiterando que un indicador formal nunca será un concepto en el sentido tradicional, que trata a la vida como una generalidad, sino que indica el sentido de ser de la vida en su especificidad. Los indicadores formales se ocupan, por lo tanto, del sentido. El término «indicación» resalta el «anuncio» 
de un sentido, y son indicativos de una "posibilidad de ser». En la medida en que la filosofía llegue a ser ontología fundamental de la facticidad, entonces los conceptos en filosofía deberán ser formalmente indicativos, anunciando el sentido de ser manifiesto. Ninguna enumeración descriptiva de caracteres generales del ente puede llegar a fijar las modalidades de ser, sino sólo la movilidad ejecutiva del cuidado que se interpreta a sí misma en sus comportamientos con relación al ente. Si nos instalamos en un determinado comportamiento, esto es, en una cierta manera de referirnos al ente y, aclaramos nuestra visión en esta referencialidad, la estructura del cuidado nos mostrará, de acuerdo con su carácter anticipativo, la manera como el ente es descubierto de acuerdo con un modo de ser. Gracias al cuidado, se pueden mantener abiertos los rasgos constitutivos de la cosa, su realidad o utilidad, pero también el campo ontológico o modo de ser del ente descubierto. Por eso, cuando Heidegger busca otra alternativa a lo dicho por Aristóteles, que «el ser se dice de muchas maneras», se ha evitado la vía rápida de una regionalización del ente y ha encontrado en la indicación formal una manera renovada para afrontar la problemática del sentido del ser y los modos de ser del ente.

\section{REFERENCIAS BIBLIOGRÁFICAS:}

de LARA, F. (2012). «El estatuto fenomenológico de la indicación formal en Heidegger», Revista Filosofía Unisinos, no. 13, pp. 15-29.

HUSSERL, E. (2013). Ideas relativas a una fenomenología pura y una filosofía fenomenológica. Libro primero: Introducción general a la fenomenología pura, México: Fondo de Cultura Económica.

HEIDEGGER, M, (2002). Interpretaciones fenomenológicas sobre Aristóteles. Indicación de la situación hermenéutica. Informe Natorp, trad. J. Adrián Escudero, Madrid: Trotta. Versión alemana: (2005). Phänomenologische Interpretationen ausgewählter Abhandlungen des Aristoteles zu Ontologie und Logik (Sommersemester 1922) Anhang: Phänomenologische Interpretationen zu Aristoteles (Anzeige der hermeneutischen Situation), Ausarbeitung für die Marburger und die Göttinger Philosophische Fakultät, GA 62, Frankfurt del Meno: Vittorio Klostermann.

(2008). Ontología. Hermenéutica de la facticidad, (Curso de verano 1923), trad. J. Aspiunza, Madrid: Alianza Editorial. Versión alemana: (1995). Ontologie. Hermeneutik der Faktizität (Sommersemester 1923), GA 63, Frankfurt del Meno: Vittorio Klostermann.

(2008b). Introducción a la investigación fenomenológica, (Curso de invierto 1923/24), trad. Juan José García Norro, Madrid: Síntesis. Versión alemana: (2006) Einführung in die phänomenologische Forschung (Wintersemester 1923/24), GA 17, editado por Friedrich-Wilhelm von Herrmann, Frankfurt del Meno: Vittorio Klostermann.

(2007). Prolegómenos para una historia del concepto de tiempo, (Curso de verano 1925), trad. Jaime Aspiunza, Madrid: Alianza. Versión alemana: 
(1994). Prolegomena zur Geschichte des Zeitbegriffs (Sommersemester 1925), GA 20, Frankfurt del Meno: Vittorio Klostermann. (2001). Ser y tiempo, trad. Jorge Eduardo Rivera C., Madrid: Trotta. Versión alemana: (1993). Sein und Zeit, Tübingen: Max Niemayer Verlang. (2007b). Los conceptos fundamentales de la metafisica. Mundo, finitud y soledad, (Curso de verano 1929), trad. Alberto Ciria, Madrid: Alianza. Versión alemana: (2004) Die Grundbegriffe der Metaphysik. Welt - Endlichkeit - Einsamkeit (Wintersemester 1929/30), GA 29/30, Frankfurt del Meno: Vittorio Klostermann.

RODRIGUEZ, R. (2007). «La idea de una interpretación fenomenológica», Devenires, vol. 8, no. 16, pp. 20-39.

VIGO, A. (2006) «La recuperación crítica de la pregunta por el ser en Heidegger», Signos Filosóficos, vol. VIII, no. 15, enero-junio, pp. 65-104.

XOLOCOTZI, A. (2004). Fenomenología de la vida fáctica. Heidegger y su camino a Ser y tiempo, México: Plaza y Valdés Editores.

Felipe Arámburo Manilla es Profesor de la Escuela de Filosofía en la Benemérita Universidad Autónoma de Puebla (BUAP) .

Líneas de Investigación:

Didáctica de la filosofía, Ontología, Filosofía antigua y Filosofía contemporánea alemana

Publicaciones recientes:

(2020). «Los problemas fundamentales de la modalización y el fenómeno del tiempo», Eikasia: revista de filosofía, 91, pp. 83-107.

(2017). «La crítica a la modernidad en Martín Heidegger y notas sobre la transmodernidad en el pensar ontohistórico», en M. C. García Aguilar y A. Aguirre Moreno (coords.), Filosofía Práctica: reflexiones desde la transmodernidad, Puebla: Benemérita Universidad Autónoma de Puebla, pp. 53-70,

Correo electrónico:fele_am@me.com 
